Goldschmidt 2021 Abstract

https://doi.org/10.7185/gold2021.5068

\title{
Isotope metallomics of aging mice
}

\author{
JEAN-DAVID MOREL ${ }^{1}$, LUCIE SAUZÉAT ${ }^{2}$ AND VINCENT \\ BALTER $^{3,4}$
}

${ }^{1}$ Ecole Polythechnique Fédérale de Lausanne

${ }^{2}$ Université Clermont Auvergne

${ }^{3}$ ENS de Lyon, Univ Lyon 1, CNRS UMR 5276, LGL-TPE

${ }^{4}$ Ecole Normale Supérieure de Lyon

Presenting Author: vincent.balter@ens-lyon.fr

The metallome, including metal concentrations and isotopic compositions, is suspected to be variable during lifetime having thus the potential for capturing some specificities of aging. Here, we have measured a suite of trace element concentrations as well as the $\mathrm{Cu}$ and $\mathrm{Zn}$ isotope compositions in organs (liver, muscle, kidney, brain and heart) at different time points $(6,16$ and 24 months) of aging mice. The mice were also characterized by phenomic, metabolomic and proteomic analysis. The results show a wealth of associations between the (isotopic) metallome and other omic layers. We show for instance that changes in hepatic $\mathrm{Cu}$ isotope compositions are correlated to age and recapitulate several aspects of the glucose/fat metabolism. For the first time for animals, we introduce metallomic as a new omic layer and adapt classic bioinformatic tools to integrate it as a new component of biological systems. 